\title{
Allopurinol Reduces Oxidative Stress in the Ovine Fetal Cardiovascular System After Repeated Episodes of Ischemia-Reperfusion
}

\author{
JAN B. DERKS, MARTIJN A. OUDIJK, HELEN L. TORRANCE, CARIN M. A. RADEMAKER, MANON J. BENDERS, \\ KARL G. ROSEN, TEREZA CINDROVA-DAVIES, AVNESH S. THAKOR, GERARD H. A. VISSER, GRAHAM J. BURTON, \\ FRANK VAN BEL, AND DINO A. GIUSSANI
}

\begin{abstract}
Departments of Perinatology [J.B.D., M.A.O., H.L.T., M.J.B., G.H.A.V., F.B.] and Pharmacology [C.M.A.R.], University Medical Centre, 1003584 CX Utrecht, The Netherlands; Department of Physiology [K.G.R.], University of Gothenburg, SE-405 30 Göteborg, Sweden; Department of Physiology, Development \& Neuroscience [T.C.-D., A.S.T., G.J.B., D.A.G.], University of Cambridge, Cambridge CB2 3EG, United Kingdom
\end{abstract}

\begin{abstract}
In complicated labor, neonatal outcome may depend not only on the extent of fetal asphyxia and acidosis but also on the effects on the fetal cardiovascular system of reactive oxygen species (ROS) generated during the ischemia-reperfusion (I/R) associated with repeated compressions of the umbilical cord. This study tested the hypothesis that maternal treatment with clinical doses of the antioxidant allopurinol in the setting of fetal asphyxia would reduce oxidative stress in the fetal cardiovascular system. The hypothesis was tested in chronically instrumented fetal sheep in late gestation by investigating the effects of maternal treatment with therapeutic doses of allopurinol or vehicle on the fetal cardiovascular system during and after episodes of I/R. The latter were produced by repeated, measured compressions of the umbilical cord. The data show that maternal treatment with allopurinol helped maintain umbilical blood flow and it reduced fetal cardiac oxidative stress after I/R of the type associated with clinically relevant acidemia and repetitive fetal heart rate decelerations. The data support the hypothesis tested and suggest that maternal treatment with allopurinol may offer plausible clinical intervention in the management of perinatal asphyxia in complicated labor. (Pediatr Res 68: 374-380, 2010)
\end{abstract}

$\mathrm{O}$ ne of the most common challenges that the fetus experiences during pregnancy, and in particular during the processes of labor and delivery, are periods of asphyxia secondary to compressions of the umbilical cord (1). Perinatal asphyxia may result in marked fetal acidosis and cardiovascular compromise with subsequent hypoxic-ischemic encephalopathy, which is predictive of developing cerebral palsy and cognitive disability later in life (2). Therefore, the prevention and management of perinatal asphyxia remain major concerns in obstetric practice today.

Received March 4, 2010; accepted June 23, 2010

Correspondence: Dino A. Giussani, Ph.D., Department of Physiology, Development \& Neuroscience, University of Cambridge, Cambridge, CB2 3EG, UK; e-mail: dag26@ cam.ac.uk

Supported by funding from the Perinatal Center Wilhelmina Children's Hospital Utrecht, the Royal Dutch Academy of Sciences (Ter Meulen Fonds), the Dutch Institute for Scientific Research (NWO), the Dutch Society of Obstetrics and Gynecology (NVOG), and the British Heart Foundation.
Repeated compressions of the umbilical cord not only induce fetal asphyxia and acidosis but also episodes of ischemia-reperfusion (I/R), promoting the generation of reactive oxygen species (ROS) such as the superoxide anion $\left(\mathrm{O}_{2}^{--}\right)$. The latter serves as a precursor of other damaging ROS, such as the hydroxyl radical $(\cdot \mathrm{OH})$ and peroxynitrite $\left(\mathrm{ONOO}^{-}\right)$. Increased $\mathrm{O}_{2}^{--}$also decreases the bio-availability of nitric oxide (NO) and, in the circulation, an increased ratio of $\mathrm{O}_{2}^{--}$: $\mathrm{NO}$ leads to endothelial dysfunction and vasoconstriction (3). Free radical generation as a result of I/R may therefore restrict blood flow in vascular beds, particularly in those, which are highly dependent on NO, such as the umbilical (4) and myocardial (5) circulations. The umbilical vascular bed and the fetal heart may therefore be particularly susceptible to repeated compressions of the umbilical cord in complicated labor as a result of oxidant stress, compounding the adverse effects of asphyxia and acidosis.

One mechanism via which ROS are generated is through the activation of the xanthine oxidase (XO) pathway. The XO inhibitor allopurinol can offer protection against I/R-induced injury not only by reducing the formation of $\mathrm{O}_{2}^{--}$but also through direct free radical scavenging properties and chelation of nonprotein bound iron (6). Consequently, the beneficial effects of allopurinol in reducing I/R damage in adult cardiology and in pediatric and adult cardiothoracic surgery have long been established $(7,8)$. However, the beneficial effects of allopurinol in the newborn period after birth asphyxia have been less well described. One study reported that treatment with allopurinol of the asphyxiated neonate improved neonatal outcome (9), but if the time-interval between I/R and treatment had been too long, or when asphyxia had been too severe, no reduction in serious morbidity or mortality was reported (10). Therefore, there has been growing interest in

\footnotetext{
Abbreviations: AUC, area under the curve; I/R, ischemia-reperfusion; MAP, maternal arterial pressure; MHR, maternal heart rate; ROS, reactive oxygen species
} 


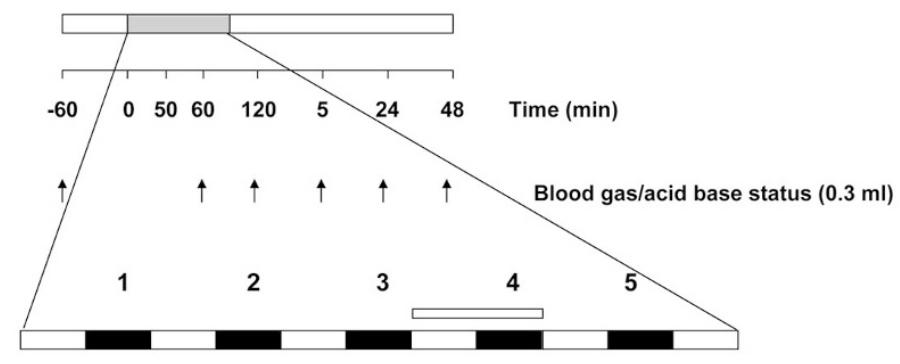

\begin{tabular}{|c|c|c|c|c|c|c|c|c|c|c|c|c|}
\hline-60 & 0 & 10 & 20 & 30 & 40 & 50 & 60 & 70 & 80 & 90 & 100 & Time (min) \\
\hline$\uparrow$ & $\uparrow$ & $\uparrow$ & $\uparrow$ & $\uparrow$ & $\uparrow$ & $\uparrow$ & $\uparrow$ & $\uparrow$ & $\uparrow$ & $\uparrow$ & $\uparrow$ & Blood gas/acid base status $(0.3 \mathrm{ml})$ \\
\hline
\end{tabular}

Figure 1. Experimental protocol. At least $5 \mathrm{~d}$ after surgery, all fetuses were submitted to an $\mathrm{I} / \mathrm{R}$ challenge (gray bar). After $1 \mathrm{~h}$ of basal recording, the I/R challenge consisted of five compressions of the umbilical cord, each of 10 min duration (black bars) with a 10-min interval. Maternal infusion with allopurinol or vehicle started $10 \mathrm{~min}$ before the fourth umbilical cord compression and finished immediately after the end of it (white bar). Fetal arterial blood samples (arrows) were taken for analysis of blood gas and metabolic status. establishing whether perinatal outcome may be improved if the window of treatment with allopurinol is initiated before birth, for instance via maternal treatment to cover the actual period of fetal asphyxia and I/R induced by recurrent umbilical cord compression in complicated labor. Maternal treatment with allopurinol crosses the placenta, it suppresses $\mathrm{O}_{2}^{--}$ production in the fetus (11) and yields therapeutic levels in the neonatal circulation (12), justifying this route of administration for preventive therapy in obstetric practice. However, virtually nothing is known about the effects of maternal treatment with allopurinol on the fetal cardiovascular system.

Therefore, this study tested the hypothesis that maternal treatment with clinical doses of allopurinol in the setting of fetal asphyxia would reduce oxidative stress in the fetal cardiovascular system. The hypothesis was tested by investigating in the chronically instrumented ovine fetus in late gestation, the effects of maternal treatment with allopurinol on the fetal cardiovascular system during and after episodes of $\mathrm{I} / \mathrm{R}$ produced by repeated, measured compression of the umbilical cord.

\section{METHODS}

All procedures were performed under the UK Animals (Scientific Procedures) Act 1986 and were approved by the Ethical Review Committee of the University of Cambridge.

Surgical preparation. Eleven Welsh Mountain Sheep were surgically instrumented for long term recording at $124 \mathrm{~d}$ of gestation (term is $\sim 145 \mathrm{~d}$ ) using strict aseptic conditions as previously described (13). Under general anesthesia (1.5-2.0\% halothane in 50:50 $\mathrm{O}_{2}: \mathrm{N}_{2} \mathrm{O}$ ), fetal arterial (inner diameter, $0.86 \mathrm{~mm}$; outer diameter, $1.52 \mathrm{~mm}$; Critchly Electrical Products, NSW, Australia) and venous (inner diameter, $0.56 \mathrm{~mm}$; outer diameter, $0.96 \mathrm{~mm}$ ) catheters were inserted. Another catheter was anchored onto the fetal hind limb for recording of the reference amniotic pressure. Two fetal ECG leads were placed s.c. on the precordium, between the apex and the left sternal border, and on the right shoulder. In addition, a transit-time flow transducer was implanted around the left umbilical artery close to the common umbilical artery inside the fetal abdomen (4SB; Transonic Systems Inc., Ithaca, NY). An inflatable occluder cuff (In Vivo Metrics, CA) was positioned around the proximal end of the umbilical cord (13). Ewes were instrumented with arterial and venous catheters placed in the left femoral artery and vein, respectively.

Postoperative care and experimental protocol. Antibiotics were administered daily to the ewe $\left(0.20-0.25 \mathrm{mg} \cdot \mathrm{kg}^{-1}\right.$ i.m. Depocillin; Mycofarm, Cambridge, UK) and fetus i.v. and into the amniotic cavity $\left(150 \mathrm{mg} \cdot \mathrm{kg}^{-1}\right.$ Penbritin; SmithKline Beecham Animal Health, Welwyn Garden City, Hertfordshire, UK). After at least $5 \mathrm{~d}$ of postoperative recovery, between 129 and $130 \mathrm{~d}$ of gestation, all fetuses were submitted to an I/R challenge, produced by $5 \times 10 \mathrm{~min}$ inflations of the cord occluder with sterile saline at 10 -min intervals (Fig. 1). Each cord compression was designed to reduce umbilical blood flow by 80 to $90 \%$ from baseline, and to lead to a progressive fall in fetal arterial $\mathrm{pH}$ to $\sim 6.95$. In five fetuses, the $\mathrm{I} / \mathrm{R}$ challenge was induced during maternal i.v. treatment with allopurinol (Sigma Chemical Co. Ltd., 20 $\mathrm{mg} \cdot \mathrm{kg}^{-1}$ maternal weight, dissolved in $20 \mathrm{~mL}$ of buffered saline and infused over a 20 -min period; $1 \mathrm{~mL} / \mathrm{min}$ ). In the remaining six fetuses, the $\mathrm{I} / \mathrm{R}$ challenge was induced during maternal infusion with buffered saline at the same rate. Infusion of either allopurinol or vehicle started $10 \mathrm{~min}$ before the fourth umbilical cord compression and finished immediately after the end of it. The dosing regimen of allopurinol was adopted from the only study that used the drug in women undergoing uncomplicated labor (12). Forty-eight hours after the end of the experimental protocol, ewes and fetuses were subjected to euthanasia (sodium pentobarbitone $200 \mathrm{mg} \cdot \mathrm{kg}^{-1}$ i.v. Pentoject; Animal Ltd., York, UK). The fetal hearts were collected and snap-frozen for measurement of pro- and antioxidant protein levels via Western blot. Hearts collected from five noninstrumented fetal sheep at 0.8 gestation served as age-matched controls.

Blood sampling regimen. Maternal and fetal arterial blood samples $(0.3$ $\mathrm{mL}$ ) were drawn into sterile syringes $1 \mathrm{~h}$ before, at $10 \mathrm{~min}$ intervals during, and for $48 \mathrm{~h}$ after the $\mathrm{I} / \mathrm{R}$ challenge to determine arterial blood gas and acid base status (Fig. 1; ABL5 Blood Gas Analyzer, Radiometer, Copenhagen, Denmark). Values for percentage saturation of $\mathrm{Hb}$ with oxygen $(\mathrm{Sat} \mathrm{Hb}$ ) were determined using a hemoximeter (OSM3; Radiometer). Blood lactate concentrations were measured by an automated analyser (Yellow Springs 2300 Stat Plus; YSI Ltd., Farnborough, UK). Fetal plasma concentrations of troponin, an index of cardiac stress, were measured at six time points during the study: at baseline ( $-60 \mathrm{~min})$, shortly before infusion with vehicle or allopurinol (50 $\mathrm{min}$ ) and after infusion at $120 \mathrm{~min}$, 5,24 , and $48 \mathrm{~h}$. Troponin was measured by chemiluminescent technology using the Beckman Unicell DxI 800 immunoassay system.

Additional paired maternal and fetal blood samples $(1 \mathrm{~mL})$ were taken in the allopurinol treated pregnancies at varying set intervals, starting at the onset of the infusion period and up to $5 \mathrm{~h}$ after the end of infusion, to compile a comprehensive serial profile of maternal and fetal plasma concentrations of allopurinol and oxypurinol (the active metabolite of allopurinol) without affecting materno-fetal concentrations of $\mathrm{Hb}$. In some animals $(n=3)$, maternal and fetal blood samples were taken during pilot experiments to address whether umbilical cord compression per se altered the concentration of allo- or oxypurinol in maternal or fetal plasma. The quantification of allopurinol and oxypurinol in both fetal and maternal plasma (HPLC) was linear between 0.5 and $25 \mathrm{mg} \cdot 1^{-1}$ with a lower limit of detection of 0.2 $\mathrm{mg} \cdot 1^{-1}$ for both compounds.

Western blotting. Tissue from the fetal left ventricle was homogenized in ice-cold buffer and centrifuged at 150,000 rpm. Aliquots were removed from the supernatant for protein quantification (BCA assay, Sigma Chemical Co., UK) and the supernatant diluted in a calculated volume of sample buffer, such that all supernatants were of equal protein concentration. Equal amounts of protein $(20-30 \mu \mathrm{g})$ from each sample were loaded onto acrylamide gels and separated by SDS-PAGE and protein from the gel was transferred onto a nitro-cellulose membrane. Once transfer was complete, membranes were stained with Ponceau to check for uniform protein loading and membranes were blocked. Membranes were incubated overnight with specific primary antibodies and after washing with a species-specific secondary antibody, followed by incubation with a substrate (ECL or ECL Plus kit, Amersham Biosciences, Bucks, UK) according to the manufacturer's instructions (see Table 1 for details). The samples were exposed to x-ray film and the levels of protein expression were quantified densitometrically as a ratio of protein loading.

Data and statistical analyses. Cardiovascular data were recorded continually at $1 \mathrm{~s}$ intervals using a computerized Data Acquisition System (Department of PDN, University of Cambridge, UK). Summary measures analysis was applied to the cardiovascular serial data to focus the number of comparisons, and areas under the curve were calculated for statistical comparison, as previously described in detail (14). The T/QRS of the fetal electrocardiogram (ECG) was analyzed using a STAN S31 (Neoventa Medical) machine. Mean values for the T/QRS ratio were calculated during baseline and at $10 \mathrm{~min}$ 
intervals during the $\mathrm{I} / \mathrm{R}$ challenge. For all variables, values are expressed as mean \pm SEM. Variables were assessed using one or two-way ANOVA with repeated measures, as appropriate. Where a significant effect was indicated, the post hoc Tukey or $t$-Newman-Keuls test was used to isolate the statistical difference (Sigma Chemical Co.-Stat; SPSS Inc., Chicago, IL). For all comparisons, statistical significance was accepted when $p<0.05$.

\section{RESULTS}

Allopurinol and oxypurinol in maternal and fetal plasma. Maternal treatment with allopurinol produced transient elevations in the concentrations of both allopurinol and oxypurinol

Table 1. Details of antibodies for Western blot analysis

\begin{tabular}{ll}
\hline \multicolumn{1}{c}{ Antibody } & \multicolumn{1}{c}{ Supplier } \\
\hline Anti-COX-2 & Cayman Chemicals (Ann Arbor, MI) \\
Anti-Hsp70 & Stressgen Bioreagents Corp. (York, UK) \\
Anti-Hsp90 & Stressgen Bioreagents Corp. (York, UK) \\
Anti-eNOS & Cell Signaling Technology (Danvers, MA) \\
Anti-MnSOD & Millipore (Watford, UK) \\
Anti-GPX & Abcam (Cambridge, UK) \\
Anti-catalase & Abcam (Cambridge, UK) \\
ECL antimouse horse radish & Amersham Biosciences (Buck., UK) \\
peroxidase-linked & \\
secondary antibody & \\
ECL antirabbit horse radish & Amersham Biosciences (Buck., UK) \\
$\quad$ peroxidase-linked & \\
secondary antibody & \\
\hline
\end{tabular}

in maternal and fetal plasma (Fig. 2). In maternal plasma, peak concentrations of allopurinol and oxypurinol occurred between 20 and $30 \mathrm{~min}$ after the start of infusion, reaching values of 47 and $17 \mathrm{mg} \cdot \mathrm{L}^{-1}$, respectively. In fetal plasma, elevations in allopurinol between 4 and $7 \mathrm{mg} \cdot \mathrm{L}^{-1}$ were within the therapeutic ranges described by Boda et al. (12), and these were maintained past $1 \mathrm{~h}$ after the start of maternal administration. Relative to allopurinol, the elevation in oxypurinol in fetal plasma was more gradual and lasted longer, reaching peak values between 1 and $1.5 \mathrm{mg} \cdot \mathrm{L}^{-1}$ at $2 \mathrm{~h}$ after the start of infusion. Compression of the umbilical cord per se did not alter maternal and fetal plasma concentrations of allopurinol and oxypurinol; these remained undetectable.

Maternal variables. Basal maternal arterial blood gas and acid base status were not different between vehicle and allopurinol pregnancies [vehicle group: $\mathrm{pH} 7.52 \pm 0.01$, partial pressure of carbon dioxide in the arterial blood $\left(\mathrm{PaCO}_{2}\right) 39 \pm$ $1 \mathrm{~mm} \mathrm{Hg}$, partial pressure of oxygen in arterial blood $\left(\mathrm{PaO}_{2}\right)$ $103 \pm 3 \mathrm{~mm} \mathrm{Hg}, \mathrm{ABE} 7.9 \pm 0.9 \mathrm{mEq} \cdot \mathrm{L}^{-1}, \mathrm{SatHb} 97 \pm 1 \%$; allopurinol group: $\mathrm{pH} 7.52 \pm 0.01, \mathrm{PacO}_{2} 37 \pm 0.4 \mathrm{~mm} \mathrm{Hg}$, $\mathrm{PaO}_{2} 100 \pm 4 \mathrm{~mm} \mathrm{Hg}, \mathrm{ABE}$ (acid/base excess) $7.0 \pm 0.9$ $\mathrm{mEq} \cdot \mathrm{L}^{-1}$, SatHb $\left.97 \pm 1 \%\right]$. These values remained unaffected from baseline throughout the experimental protocol. Basal maternal arterial blood pressure (MAP) and heart rate


Figure 2. Maternal and fetal plasma allopurinol and oxypurinol. The data show the serial profile of plasma concentrations of allopurinol and oxypurinol in maternal (open symbols, panels A and B) and fetal (solid symbols, panels C and D) plasma after maternal treatment with allopurinol. Paired maternal and fetal blood samples $(1 \mathrm{~mL})$ were taken in the allopurinoltreated pregnancies at varying set intervals, starting at the onset of the allopurinol infusion period (time point $50 \mathrm{~min}$ in Fig. 1) and for $5 \mathrm{~h}$ thereafter.

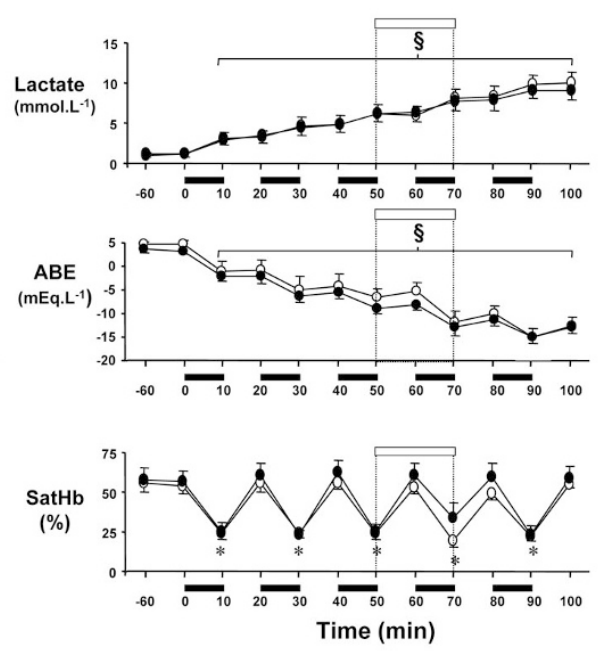

Figure 3. Fetal blood gas and acid base status. Data are the mean \pm SEM for fetal blood gas and acid base status in samples taken before, during and after each umbilical cord compression. In five fetuses (-), the I/R challenge was induced during maternal i.v. treatment with allopurinol. In the other six fetuses $(O)$, the $I / R$ challenge was induced during maternal infusion with buffered saline at the same rate. Significant differences within groups are $* p<0.05$ vs immediately preceding baseline; $\S p<0.05 v s$ -60 min sample (two-way RM ANOVA with post hoc $t$-Newman-Keuls test). 
(MHR) were not different between groups (vehicle: MAP $104 \pm 10$ mm Hg, MHR $97 \pm 6$ bpm; allopurinol: MAP $99 \pm$ $5 \mathrm{~mm} \mathrm{Hg}$, MHR $91 \pm 5 \mathrm{bpm})$. Although MAP remained unaffected from baseline in both groups, there was a transient increase from baseline in MHR to a maximum of $156 \pm 20$ bpm at $52 \mathrm{~min}$ after the onset of infusion in ewes treated with allopurinol $(p<0.05)$.

Fetal arterial blood gas, metabolic status, and survival. Compressions of the umbilical cord produced transient episodes of hypoxia and hypercapnia of similar magnitude in both vehicle and allopurinol groups (Fig. 3). Repeated umbilical cord compression also led to a gradual decline in fetal basal $\mathrm{pH}$ and $\mathrm{ABE}$ and a gradual increase in fetal basal lactate concentrations. Again, the magnitudes of these changes were similar between groups (Fig. 3). By the end of the fifth compression, fetal $\mathrm{pH}$ was reduced from $7.36 \pm 0$ at baseline to $6.97 \pm 0.03$ versus $7.36 \pm 0$ to $6.97 \pm 0.03$ (both groups $p<0.05)$.

All fetuses in the pregnancies treated with allopurinol survived the I/R challenge and they were in good physiologic condition $48 \mathrm{~h}$ after the end of the study protocol, with values representing fetal arterial blood gas and metabolic status similar to those measured before I/R. In contrast, one fetus from the vehicle treated pregnancies died $3 \mathrm{~h}$ after the end of the I/R challenge as a result of cardiovascular collapse. Values for cardiovascular data in this fetus after the end of the I/R challenge have been excluded from the data analysis.

Fetal cardiovascular variables. Mean values for basal fetal arterial blood pressure and basal fetal heart rate were similar between vehicle and allopurinol groups $(55.4 \pm 3.6 \mathrm{~mm} \mathrm{Hg}$ versus $53.8 \pm 3.6$ and $164 \pm 5$ versus $163 \pm 10 \mathrm{bpm}$, respectively). Values for these variables during the I/R experimental protocol are shown as percentage changes from baseline in Fig. 4. Compression of the umbilical cord led to rapid transient falls in fetal heart rate and rapid transient increases in fetal arterial blood pressure. The magnitude and pattern of these changes were similar between vehicle and allopurinol pregnancies until the onset of maternal treatment with allopurinol. Maternal treatment with allopurinol led to an increase in values for fetal heart rate calculated before the fourth $(185 \pm 17$ versus $193 \pm 13 \mathrm{bpm})$ and fifth $(193 \pm 14$ versus $210 \pm 12 \mathrm{bpm}$ ) compression [control versus allopurinol, area under the curve (AUC), $p<0.05]$. After the I/R challenge, fetal arterial blood pressure remained significantly below and fetal heart rate remained significantly above basal values in both groups of pregnancies until the end of the fifth recording period (Fig. 4). The pattern and magnitude of the fall in fetal arterial blood pressure postI/R were similar between the groups. In contrast, postI/R, while the increase in fetal heart rate was mild and sustained in control pregnancies, it was markedly and transiently exacerbated in allopurinol pregnancies, showing a $53 \%$ increase at $110 \mathrm{~min}$ after the onset of maternal treatment with allopurinol.

Mean values for basal umbilical blood flow were similar between vehicle and allopurinol groups (138 \pm 19 versus $135 \pm 28 \mathrm{~mL} \cdot \mathrm{min}^{-1}$, respectively). Umbilical blood flow was similarly reduced by 80 to $90 \%$ from baseline during each compression period in both groups (Fig. 4). After the I/R
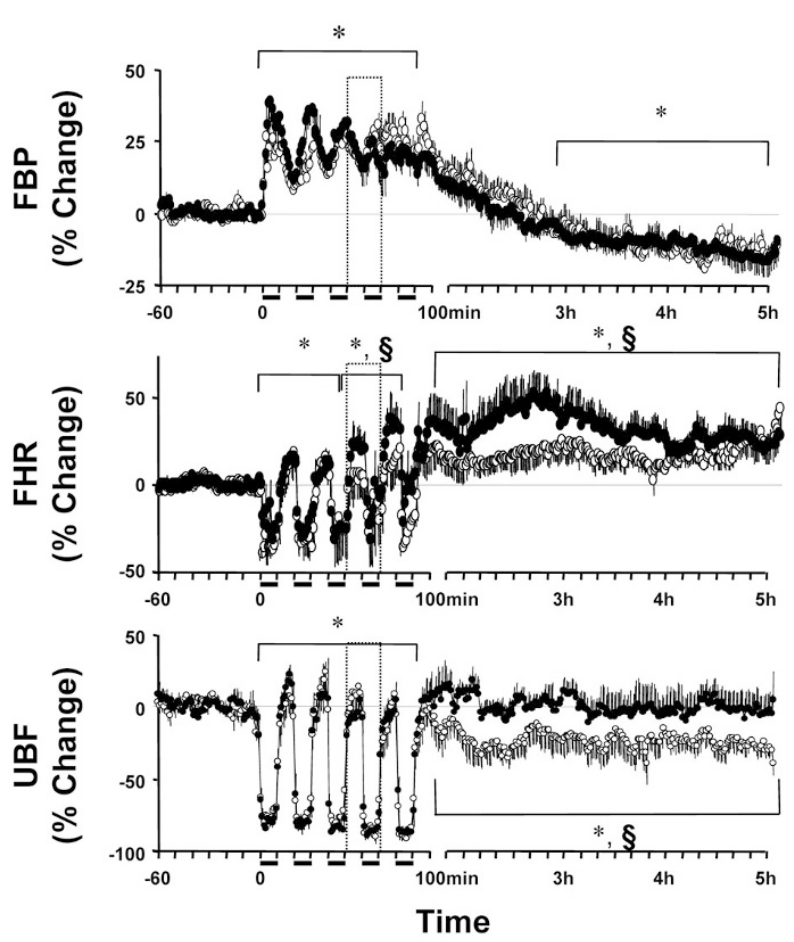

Figure 4. Fetal cardiovascular variables. Data are the mean \pm SEM of the percent change from mean baseline calculated every minute in six allopurinol pregnancies (O) and in buffered saline six pregnancies (O). Significant differences are comparisons of AUC: ${ }^{*} p<0.05$ vs baseline $1 \mathrm{~h}$ period; $\S p<$ 0.05 , allopurinol $v s$ saline (two-way RM ANOVA with $t$-Newman-Keuls test).

challenge, umbilical blood flow returned to basal values within $5 \mathrm{~min}$ in pregnancies treated with allopurinol. In marked contrast, in pregnancies treated with vehicle, umbilical blood flow remained significantly depressed from basal values until the end of the recording period, $5 \mathrm{~h}$ after the start of the challenge (Fig. 4).

In the vehicle group, there was a significant increase in the fetal T/QRS ratio during each of the five compressions of the umbilical cord (Figs. 5 and 6). In addition, values for the fetal T/QRS ratio in this group remained significantly elevated from baseline immediately after the fourth and fifth umbilical cord compression (Fig. 6). In the allopurinol group, the fetal T/QRS was also significantly elevated during each of the five umbilical cord compressions. However, the values for T/QRS achieved in this group were significantly lower than those of fetuses in the vehicle group after treatment (Fig. 6).

Basal values for concentrations of troponin in fetal plasma were similar in vehicle and allopurinol groups (Fig. 7). Although fetal plasma troponin increased significantly at $20 \mathrm{~min}$ (time point $120 \mathrm{~min}$ ) and $5 \mathrm{~h}$ after the I/R challenge in vehicle treated pregnancies, these increases were prevented in allopurinol treated pregnancies (Fig. 7).

Cardiac pro- and antioxidant proteins. Analysis by Western blot revealed a significant increase in the protein expression of COX-2, eNOS, and Hsp90 in hearts isolated from fetuses subjected to I/R treated with vehicle relative to hearts isolated from control fetuses (Fig. 8). Maternal treatment with allopurinol reverted the increase in fetal cardiac COX-2, eNOS but not Hsp90. Similarly, levels of the antioxidant 


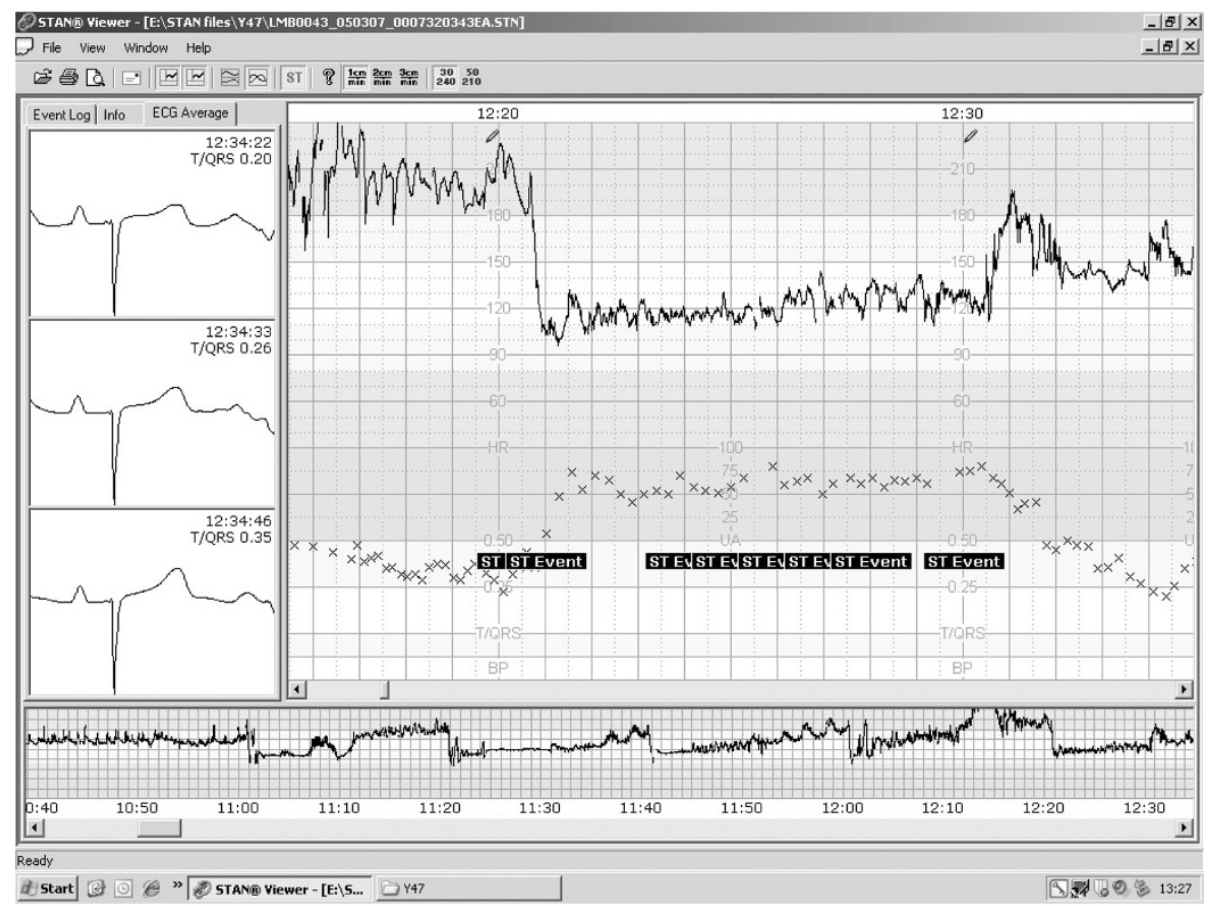

Figure 5. Fetal ECG wave form after umbilical cord compression. Example of an ECG wave form trace and associated STAN event obtained before and after the onset of an umbilical cord occlusion event in a late gestation sheep fetus (Y47). Umbilical cord compression started at 12:20.

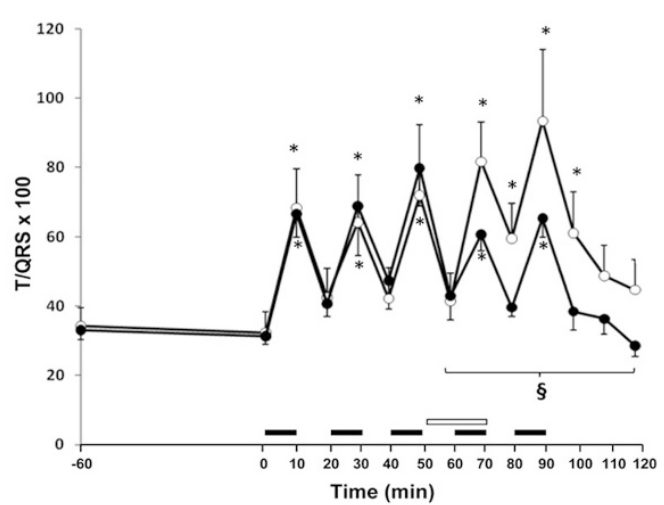

Figure 6. Fetal T/QRS ratio. Data are mean \pm SEM of the fetal T/QRS analyzed using a STAN S31 (Neoventa Medical) machine in six allopurinol pregnancies $(\bullet)$ and in six buffered saline pregnancies $(\bigcirc)$. Significant differences are $* p<0.05 v s$ baseline; $\S p<0.05$, allopurinol $v s$ saline AUC (two-way RM ANOVA with $t$-Newman-Keuls test).

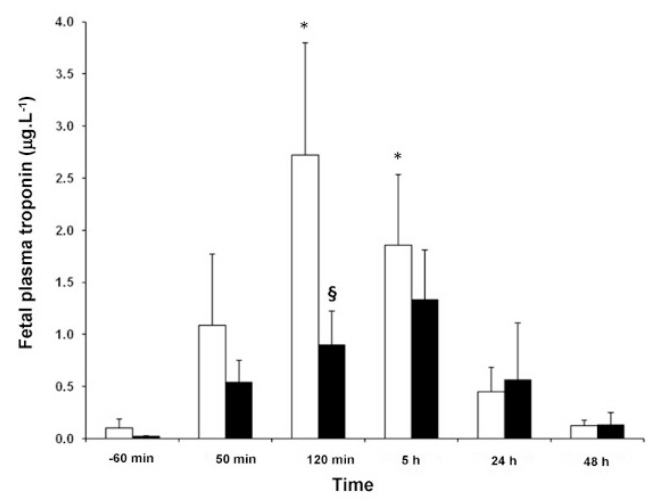

Figure 7. Fetal plasma troponin. Data are mean \pm SEM of troponin measured in circulating fetal plasma in six allopurinol (ם) and in six buffered saline pregnancies $(\square)$. Significant differences are $* p<0.05$ vs baseline; $\S p<0.05$, allopurinol $v s$ saline (two-way RM ANOVA with $t$-NewmanKeuls test). proteins $\mathrm{mnSOD}$ and GPX were significantly decreased in hearts isolated from fetuses subjected to $\mathrm{I} / \mathrm{R}$ during vehicle relative to hearts isolated from control fetuses (Fig. 8). Maternal treatment with allopurinol partially reduced the decrease in fetal cardiac mnSOD and GPX.

\section{DISCUSSION}

The data show that in the late gestation ovine fetus repeated, intermittent, measured compression of the umbilical cord designed to reduce umbilical blood flow by 80 to $90 \%$ of basal values led to repeated fetal bradycardia and progressive fetal acidemia of human clinical relevance (mean \pm SEM: $6.97 \pm$ 0.03 in both vehicle and allopurinol treated pregnancies). After the I/R challenge, umbilical blood flow in untreated pregnancies remained significantly depressed from basal values until the end of the recording period, $5 \mathrm{~h}$ after the start of the challenge. In addition, one fetus in this group died $3 \mathrm{~h}$ after the end of the I/R challenge. In marked contrast, in pregnancies treated with allopurinol, umbilical blood flow was restored to basal values within $5 \mathrm{~min}$ after $\mathrm{I} / \mathrm{R}$ and all fetuses in this group survived the challenge. These data suggest that $I / R$ does compound the effects on the fetus of asphyxia and acidosis by restricting umbilical blood flow to extents that may lead to fetal death. Episodes of I/R may increase the ratio of vascular $\mathrm{O}_{2}^{-}: \mathrm{NO}$ in the umbilical vascular bed. Maternal treatment with allopurinol may thus increase the bioavailability of NO, protecting against increases in umbilical vascular resistance and better maintaining umbilical blood flow. Further, the data show that the physiologic mechanism underlying the effects of I/R on the umbilical vascular bed is, in part, due to the activation of the XO pathway. Accordingly, $\mathrm{XO}$ is known to be present in the human placental vascular bed (15). Second, placental XO activity is up-regulated during labor (16). Third, ROS, generated by hypoxanthine and XO, poten- 

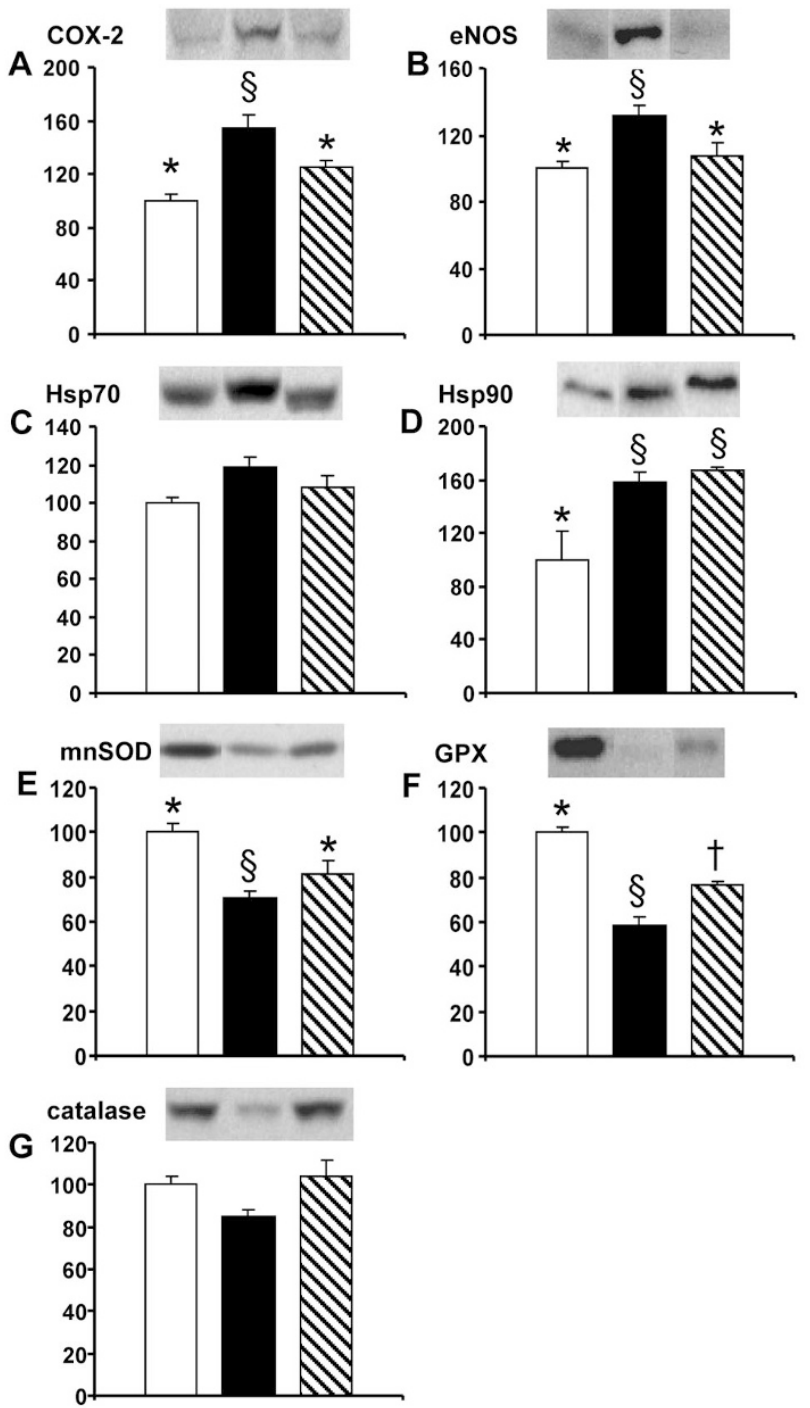

Figure 8. Pro- and antioxidant proteins in the fetal heart. Data are the mean \pm SEM of pro-oxidant and antioxidant proteins expressed as a percentage of control in fetal hearts in pregnancies, which received during the $\mathrm{I} / \mathrm{R}$ challenge either maternal i.v. treatment with allopurinol $(n=5, \mathbb{\mathbb { S }})$ or buffered saline $(n=5, \boldsymbol{\square})$, and in 5 age-matched control fetuses $(\square)$. Different symbols are significantly different $p<0.05$ (one-way ANOVA with Tukey test).

tiated the vascular tension in the human umbilical artery, and pretreatment with the NO synthase inhibitor NG-methyl-Larginine (LNMA) significantly attenuated the vasospasmic effect of ROS. The latter suggests that a component of the constrictor effects of XO-induced ROS on the umbilical vascular bed may be mediated specifically by decreasing the bioavailability of $\mathrm{NO}(3)$.

In this study, any effects on fetal cardiac compromise of $\mathrm{I} / \mathrm{R}$ with and without maternal treatment with allopurinol were assessed by monitoring changes in the fetal T/QRS ratio (17), measurement of concentrations of troponin in circulating fetal plasma, and alterations in cardiac pro- and antioxidant proteins. It is established that fetal asphyxia promotes elevations of the ST segment and an increase in the height of the T-wave in the fetal ECG, as a result of a surge in adrenaline and myocardial glycogenolysis (18). An increase in the T/QRS ratio and in circulating concentrations of troponin are also established measures of enhanced myocardial performance, workload, and damage $(19,20)$. In this study, maternal treatment with allopurinol reduced the increments from baseline in the fetal T/QRS ratio during compression of the umbilical cord posttreatment and it also reduced the increment in fetal plasma troponin concentrations after the $\mathrm{I} / \mathrm{R}$ challenge, results consistent with a protective effect of allopurinol on fetal cardiac workload during and immediately after asphyxia. Several mechanisms could be responsible for these protective effects. Inkeeping with this line of thinking, studies of heart failure have reported that allopurinol resulted in decreased myocardial oxygen consumption and improved mechanical efficiency in pacing induced heart failure (21).

Further data in the article show that episodes of I/R led to increased expression of oxidant proteins and decreased expression of antioxidant proteins in the fetal heart, findings consistent with the induction of oxidative stress in the fetal heart. Hearts isolated from fetuses subjected to I/R showed a significant increase in the protein expression of COX-2, eNOS, and Hsp90 relative to control fetuses. COX-2 has been shown to be induced in neonatal rat cardiomyocytes in response to oxidative stress (22) and xanthine oxidoreductase has been reported to be a regulator of COX-2 expression (23). Taken together these findings suggest that allopurinol prevents the induction of COX-2 through XO inhibition. Although constitutively expressed, eNOS activity is regulated by a number of factors, including the molecular chaperone Hsp90. The data in this study show that episodes of $I / R$ increase eNOS and Hsp90 in the fetal heart, suggesting that umbilical cord compression in complicated labor may increase the susceptibility of eNOS to become uncoupled and produce further ROS. Interestingly, eNOS up-regulation after $\mathrm{I} / \mathrm{R}$ did not occur in fetuses whose mothers received allopurinol treatment. Decreased production of ROS secondary to activation of the XO pathway in allopurinol treated pregnancies may better maintain the bioavailability of NO, thereby preventing any need for eNOS up-regulation. Further, excess NO is known to decrease eNOS expression, a negative feedback effect demonstrated both in vivo and in the vascular tissues of rats after administration of an NO donor (24).

Manganese superoxide dismutase, catalase, and glutathione peroxidase are powerful antioxidant enzymes that contribute to the ability of the cell to quench ROS. Glutathione is known to play a far more important role as a hydrogen peroxide scavenger, particularly in the heart because its biologic activity is much greater than that of catalase (23). The diminished decrease in the fetal cardiac expression of antioxidant enzymes in allopurinol treated pregnancies after I/R again suggests reduced ROS generation, alleviating the consumption of antioxidant defenses in the fetal heart.

In conclusion, in vivo and molecular data in this study strongly support the hypothesis tested that maternal treatment with clinical doses of allopurinol in the setting of fetal asphyxia reduces oxidative stress in the fetal cardiovascular system. The data suggest that maternal treatment with allopurinol may offer plausible clinical intervention in the management of perinatal asphyxia in complicated labor. 
Acknowledgments. We thank Mr Scott Gentle and Mrs Sue Nicholls for their help with the animal maintenance.

\section{REFERENCES}

1. Clapp JF, Peress NS, Wesley M, Mann LI 1988 Brain damage after intermittent partial cord occlusion in the chronically instrumented fetal lamb. Am J Obstet Gynecol 159:504-509

2. Frasch MG, Mansano RZ, McPhaul L, Gagnon R, Richardson BS, Ross MG 2009 Measures of acidosis with repetitive umbilical cord occlusions leading to fetal asphyxia in the near-term ovine fetus. Am J Obstet Gynecol 200:200.e1-200.e7

3. Chen K, Keaney J 2004 Reactive oxygen species-mediated signal transduction in the endothelium. Endothelium 11:109-121

4. Chang JK, Roman C, Heymann MA 1992 Effect of endothelium-derived relaxing factor inhibition on the umbilical-placental circulation in fetal lambs in utero. Am J Obstet Gynecol 166:727-734

5. Thornburg KL, Reller MD 1999 Coronary flow regulation in the fetal sheep. Am J Physiol 277:R1249-R1260

6. Berry CE, Hare JM 2004 Xanthine oxidoreductase and cardiovascular disease: molecular mechanisms and pathophysiological implications. J Physiol 555:589-606

7. Castelli P, Condemi AM, Brambillasca C, Fundaro P, Botta M, Lemma M, Vanelli P, Santoli C, Gatti S, Riva E 1995 Improvement of cardiac function by allopurinol in patients undergoing cardiac surgery. J Cardiovasc Pharmacol 25:119-125

8. Clancy RR, McGaurn SA, Goin JE, Hirtz DG, Norwood WI, Gaynor JW, Jacobs ML, Wernovsky G, Mahle WT, Murphy JD, Nicolson SC, Steven JM, Spray TL 2001 Allopurinol neurocardiac protection trial in infants undergoing heart surgery using deep hypothermic circulatory arrest. Pediatrics 108:61-70

9. Van Bel F, Shadid M, Moison RM, Dorrepaal CA, Fontijn J, Monteiro L, van de Bor M, Berger HM 1998 Effect of allopurinol on postasphyxial free radical formation, cerebral hemodynamics, and electrical brain activity. Pediatrics 101:185-193

10. Benders MJ, Bos AF, Rademaker CM, Rijken M, Torrance HL, Groenendaal F, van Bel F 2006 Early postnatal allopurinol does not improve short-term outcome after severe birth asphyxia. Arch Dis Child Fetal Neonatal Ed 91:F163-F165

11. Masaoka N, Nakajima Y, Hayakawa Y, Ohgame S, Hamano S, Nagaishi M, Yamamoto T 2005 Transplacental effects of allopurinol on suppression of oxygen free radical production in chronically instrumented fetal lamb brains during intermittent umbilical cord occlusion. J Matern Fetal Neonatal Med 18:1-7

12. Boda D, Nemeth I, Kiss P, Orvos H 1999 Treatment of mothers with allopurinol to produce therapeutic blood levels in newborns. Prenat Neonatal Med 4:130-134

13. Thakor AS, Giussani DA 2005 Calcitonin gene-related peptide contributes to the umbilical haemodynamic defence response to acute hypoxaemia. J Physiol 563:309-317

14. Matthews JN, Altman DG, Campbell MJ, Royston P 1990 Analysis of serial measurements in medical research. BMJ 300:230-235

15. Many A, Westerhausen-Larson A, Kanbour-Shakir A, Roberts JM 1996 Xanthine oxidase/dehydrogenase is present in human placenta. Placenta 17:361-365

16. Many A, Roberts JM 1997 Increased xanthine oxidase during labour-implications for oxidative stress. Placenta 18:725-726

17. Amer-Wåhlin I, Hellsten C, Norén H, Hagberg H, Herbst A, Kiellmer I, Lilja H, Lindoff C, Månsson M, Mårtensson L, Olofsson P, Sundström A, Marsál K 2001 Cardiotocography only versus cardiotocography plus ST analysis of fetal electrocardiogram for intrapartum fetal monitoring: a Swedish randomised trial. Lancet 358:534-538

18. Hökegård KH, Eriksson BO, Kjellmer I, Magno R, Rosén KG 1981 Myocardial metabolism in relation to electrocardiographic changes and cardiac function during graded hypoxia in the fetal lamb. Acta Physiol Scand 113:1-7

19. Rosén KG, Luzietti R 1994 The fetal electrocardiogram: ST waveform analysis during labour. J Perinat Med 22:501-512

20. Adams JE III, Bodor GS, Davila-Roman VG, Delmez JA, Apple FS, Ladenson JH, Jaffe AS 1993 Cardiac troponin I. A marker with high specificity for cardiac injury. Circulation 88:101-106

21. Cappola TP, Kass DA, Nelson GS, Berger RD, Rosas GO, Kobeissi ZA, Marbán E, Hare JM 2001 Allopurinol improves myocardial efficiency in patients with idiopathic dilated cardiomyopathy. Circulation 104:2407-2411

22. Piscopo P, Bernardo A, Calamandrei G, Venerosi A, Valanzano A, Bianchi D, Confaloni A, Minghetti L 2008 Altered expression of cyclooxygenase-2, presenilins and oxygen radical scavenging enzymes in a rat model of global perinatal asphyxia. Exp Neurol 209:192-198

23. Ohtsubo T, Rovira II, Starost MF, Liu C, Finkel T 2004 Xanthine oxidoreductase is an endogenous regulator of cyclooxygenase-2. Circ Res 95:1118-1124

24. Vaziri ND, Wang XQ 1999 cGMP-mediated negative-feedback regulation of endothelial nitric oxide synthase expression by nitric oxide. Hypertension 34:1237-1241 